
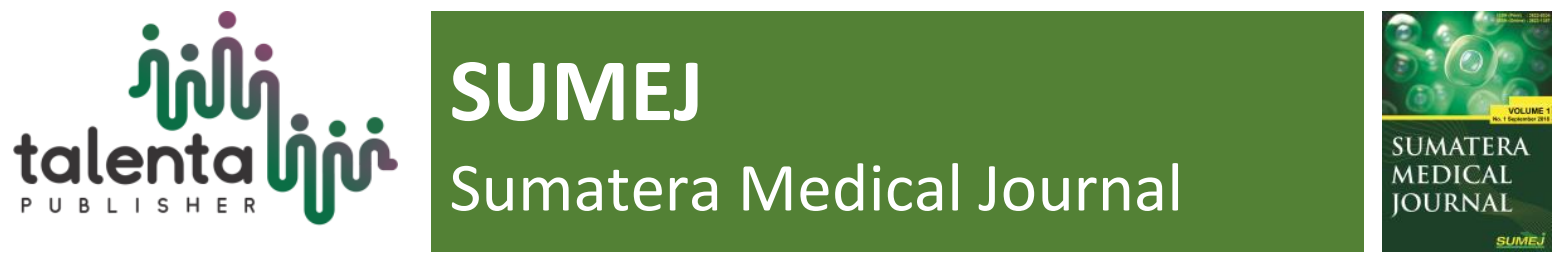

\title{
Risk Factors Analysis of Premature Canities in Medical Students of Universitas Sumatera Utara Class 2016-2018
}

\author{
E. Z. S. Lubis ${ }^{1 *}$, N. K. Jusuf ${ }^{2}$ \\ ${ }^{1)}$ Faculty of Medicine, Universitas Sumatera Utara, ${ }^{2}$ Department of Dermatology and Venereology, \\ Universitas Sumatera Utara, Medan, Indonesia
}

\begin{abstract}
Background. Premature canities is a terminology to describe a premature graying of hair before the age of 25 in Asians. The ethiopathogenesis of premature canities is still not fully understood, but there are risk factors associated to premature canities such as gender, family history, obesity, smoking, alcohol consumption, other disease, micronutrients deficiencies. Aims: to evaluate risk factors that influence the incidence of premature canities in medical students of University of Sumatera Utara class 2016-2018. Methods: This research is an analytic research with the number of subjects are 80 respondents using bivariate and multivariate analysis using logistic regression test. Results: of the 80 respondents, 57 respondents $(71,2 \%)$ had premature canities. Based on bivariate analysis, it was obtained $\mathrm{p}$ value $\leq 0,05$ for family history and stress, Based on multiavariate analysis, it was obtained $p$ value $\leq 0,05$ for family history ( $p$ value $=0,000$ ), which means family history is the most dominants factors for premature canities. Conclusion: there is a correlation between premature canities and family history and stress and Family history is the most dominant risk factors that associated to premature canities in medical students of University of Sumatera Utara class 2016-2018.
\end{abstract}

Keyword: Premature Canities, Risk Factors

Received 14 December 2019| Revised 14 January 2020 | Accepted 19 January 2020

\section{Introduction}

Hair is a keratinized epithelial cell structure that acts as a protector of scalp from sunlight exposure. Hair also plays an important role on someone's physical appearance and selfperception. Therefore, premature canities gives a significant impact on someone's interpersonal life and acceptance in the community [1].

In humans, hair pigmentation depends entirely on the amount and distribution of melanin pigments produced by melanocytes in the hair bulb. Melanogenic activity occurs during the anagen phase of the hair growth cycle. This process plays an important role in the incidence of

\footnotetext{
*Corresponding author at: Faculty of Medicine, Universitas Sumatera Utara, Medan, Indonesia 
premature canities. Premature canities is the result of functional decline in melanocytes which results in whitening hair [2].

Hair graying is one of the natural aging processes. However, in fact, many children who are still young but already have hair graying. Although it is generally not a medical problem, it greatly concerns manypeople for aesthetic reasons [3]. Premature canities has significant adverse effects on the social stigma, discrimination, and difficulties in interaction [2]. In previous studies, premature canities also affects the quality of life for each individual [4].

Premature hair graying or premature canities is the result of decreasing tyrosinase activity in melanocytes in the hair bulb [5]. Premature canities occurs regardless of sex, race and ethnicity, and can be caused by reduced progressive melanin pigment production. Although there is no universal agreement for diagnosing premature canities, Premature canities can be diagnosed if hair graying appears before the age of 20 in Caucasians, 25 in Asians, and 30 in Africans [6].

The ethiopathogenesis of premature canities is still not fully understood, however, in various studies there are several factors that asssociated to the incidence of premature canities, such as oxidative stress, comorbidities [7], family history, alcohol consumption, educational status, hair loss, age, height, weight and stress are related to premature canities [8]. Smoking and obesity are also known to associate with the incidence of premature canities [3]. Ferritin serum levels, vitamin B12, and HDL-C levels are also widely associated with premature canities (Chakrabarty et al., 2016), likewise serum levels of iron, zinc and copper ions that affect premature canities [9].

\section{Methodology}

\subsection{Study Design}

This research is an analytic research using cross sectional study approach which analyzed risk factors associated to premature canities.

\subsection{Setting and sample}

This research was conducted at the Faculty of Medicine, Universitas Sumatera Utara on September-November 2019 with the number of research subject are 80 respondents using a structured questionnaire to obtain risk factors which are associated with the incidence of premature canities. Bivariate and multivariate analysis using logistic regression test is used to examine the correlation between risk factors and premature canities. 


\section{Result}

The frequency distribution of premature canities in medical students of Faculty of medicine Universitas Sumatera Utara class 2016-2018 are presented in Table 1.

Table 1.Frequency distribution of research subject characteristics

\begin{tabular}{|c|c|c|}
\hline Variable & $\mathbf{N}$ & $\%$ \\
\hline \multicolumn{3}{|l|}{ Gender } \\
\hline Man & 37 & 46,3 \\
\hline Woman & 43 & 53,8 \\
\hline \multicolumn{3}{|l|}{ Family History } \\
\hline Father & 27 & 33,8 \\
\hline Mother & 12 & 15 \\
\hline Siblings & 13 & 16,3 \\
\hline None & 28 & 35 \\
\hline \multicolumn{3}{|l|}{ BMI } \\
\hline Normoweight & 23 & 28,8 \\
\hline Overweight & 29 & 36,3 \\
\hline Obese & 28 & 35 \\
\hline \multicolumn{3}{|l|}{ Diet } \\
\hline \multicolumn{3}{|l|}{ Vegetables intake/day } \\
\hline Yes & 39 & 48,8 \\
\hline No & 41 & 51,3 \\
\hline \multicolumn{3}{|l|}{ Meats intake/day } \\
\hline Yes & 33 & 41,3 \\
\hline No & 47 & 58,8 \\
\hline \multicolumn{3}{|l|}{ Milks intake/day } \\
\hline Yes & 34 & 42,5 \\
\hline No & 46 & 57,5 \\
\hline \multicolumn{3}{|l|}{ Comorbidities } \\
\hline None & 57 & 71,3 \\
\hline Anemia & 4 & 5 \\
\hline Others (rhinitis, allergies) & 19 & 23,8 \\
\hline \multicolumn{3}{|l|}{ Smoking } \\
\hline Never & 79 & 98,8 \\
\hline 1-5 sticks/day & 1 & 1,3 \\
\hline \multicolumn{3}{|l|}{ Alcohol } \\
\hline Never & 79 & 98,8 \\
\hline 1-2 times/month & 1 & 1,3 \\
\hline \multicolumn{3}{|l|}{ Stress } \\
\hline Mild & 11 & 13,8 \\
\hline Moderate & 54 & 67,5 \\
\hline Severe & 15 & 18,8 \\
\hline \multicolumn{3}{|l|}{ Premature Canities } \\
\hline None & 23 & 28,8 \\
\hline$<10$ & 34 & 42,5 \\
\hline $10-100$ & 22 & 27,5 \\
\hline$>100$ & 1 & 1,3 \\
\hline Total & 80 & 100 \\
\hline
\end{tabular}

Table 1 show that based on the characteristics above, of the 80 respondents, 57 respondents $(71,2 \%)$ had premature canities. The most incidence of premature canities were found in woman as 43 people $(53,8 \%)$, father's history with premature canities as 27 people $(33,8 \%)$, overweight as 29 people (36,3\%), low daily vegetables intake as 41 people $(51,3 \%)$, low daily 
meats intake as 47 people $(58,8 \%)$, low daily milks intake as 46 people $(57,5 \%)$, no comorbidities as 57 people (71,3\%), never smoking and acohol consumptions as 79 people $(98,8 \%)$, and moderate stress as 54 people $(67,5 \%)$

Table 2.Relationship between gender and premature canities

\begin{tabular}{lccccccc}
\hline & \multicolumn{8}{c}{ Premature Canities } & \multirow{2}{*}{$\begin{array}{c}\text { P } \\
\text { Variable }\end{array}$} & \multicolumn{2}{c}{ Yes } & \multicolumn{2}{c}{ No } & \multicolumn{2}{c}{ Total } & value \\
\cline { 2 - 7 } & $\mathrm{N}$ & $\%$ & $\mathrm{n}$ & $\%$ & $\mathrm{n}$ & $\%$ & \\
\hline Gender & & & & & & & \\
Man & 28 & 49,1 & 9 & 39,1 & 37 & 46,3 & 0,573 \\
Woman & 29 & 50,9 & 14 & 60,9 & 43 & 53,8 & \\
\hline Total & 57 & 100 & 23 & 100 & 80 & 100 & \\
\hline
\end{tabular}

Table 2 show that in this study, woman was found to have premature canities as 29 people $(50,9 \%)$ and man as 28 people $(49,1 \%)$. Based on statistical tests, $p$ value $=0,573$ was obtained so there was no a significant relationship between gender and premature canities $(p>0,05)$.

Table 3.Relationship between family history and premature canities

\begin{tabular}{|c|c|c|c|c|c|c|c|}
\hline \multirow{3}{*}{ Variable } & \multicolumn{6}{|c|}{ Premature Canities } & \multirow{3}{*}{$P$ value } \\
\hline & \multicolumn{2}{|c|}{ Yes } & \multicolumn{2}{|c|}{ No } & \multicolumn{2}{|c|}{ Total } & \\
\hline & $\mathrm{n}$ & $\%$ & $\mathrm{n}$ & $\%$ & $\mathrm{n}$ & $\%$ & \\
\hline \multicolumn{8}{|c|}{ Family History } \\
\hline Father & 23 & 40,4 & 4 & 17,4 & 27 & 33,8 & \multirow{4}{*}{0,000} \\
\hline Mother & 12 & 21,1 & 0 & 0,0 & 12 & 15,0 & \\
\hline Siblings & 12 & 21,1 & 1 & 4,3 & 13 & 6,3 & \\
\hline None & 10 & 17,5 & 18 & 78,3 & 28 & 35,0 & \\
\hline Total & 57 & 100 & 23 & 100 & 80 & 100 & \\
\hline
\end{tabular}

Table 3 show that in this study, the incidence of premature canities mostly occurred to the respondents who have father's history of having premature canities as many as 23 people $(40,4 \%)$, followed by mother and sibling's history of premature canities as 12 people $(21,1 \%)$, while respondents who don't have a family history of premature canities as 10 people (17,5\%). Based on statistical tests, $\mathrm{p}$ value $=0,000$ was obtained so there was a significant relationship between gender and premature canities $(\mathrm{p}<0,05)$.

Table 4.Relationship between body mass index and premature canities

\begin{tabular}{llllllll}
\hline \multirow{3}{*}{ Variables } & \multicolumn{6}{c}{ Premature Canities } & \multirow{2}{*}{ P value } \\
\cline { 2 - 7 } & \multicolumn{2}{c}{ Yes } & \multicolumn{1}{c}{ No } & \multicolumn{2}{c}{ Total } & \\
\cline { 2 - 7 } & $\mathrm{n}$ & $\%$ & $\mathrm{n}$ & $\%$ & $\mathrm{n}$ & $\%$ \\
\hline
\end{tabular}




\begin{tabular}{lcccccccc}
\hline \multicolumn{1}{c}{ BMI } & & & & & & & \\
Normoweight & 14 & 24,6 & 9 & & 39,1 & 23 & 28,8 & 0,104 \\
Overweight & 19 & 33,3 & 10 & 43,5 & 29 & 36,3 & \\
Obese & 24 & 42,1 & 4 & 17,4 & 28 & 35,0 & \\
\hline Total & 57 & 100 & 23 & 100 & 80 & 100 & \\
\hline
\end{tabular}

Table 4 show that the respondents who had normoweight and premature canities were 14 people $(24,6 \%)$, overweight as 19 people $(33,3 \%)$ and obese as many as 24 people $(42,1 \%)$. Based on statistical tests, $p$ value $=0,104$ was obtained so there was no a significant relationship between body mass index and premature canities $(\mathrm{p}>0,05)$.

Table 5.Relationship between daily vegetables intake and premature canities

\begin{tabular}{|c|c|c|c|c|c|c|c|}
\hline \multirow{3}{*}{ Variable } & \multicolumn{6}{|c|}{ Premature Canities } & \multirow{3}{*}{$P$ value } \\
\hline & \multicolumn{2}{|c|}{ Yes } & \multicolumn{2}{|c|}{ No } & \multicolumn{2}{|c|}{ Total } & \\
\hline & $\mathrm{n}$ & $\%$ & $\mathrm{n}$ & $\%$ & $\mathrm{n}$ & $\%$ & \\
\hline Vegetables intake/daily & & & & & & & 0,104 \\
\hline Yes & 24 & 42,1 & 15 & 65,2 & 39 & 48,8 & \\
\hline No & 33 & 57,9 & 8 & 34,8 & 41 & 51,3 & \\
\hline Total & 57 & 100 & 23 & 100 & 80 & 100 & \\
\hline
\end{tabular}

Table 5 show that the most incidents of premature canities occurred to the respondents who had low daily vegetables intake as many as 33 people $(57,9 \%)$ while the respondents who had vegetables intake everyday as 24 people $(42,1 \%)$. Based on statistical tests, $p$ value $=0,104$ was obtained so there was no a significant relationship between daily vegetables intake and premature canities $(\mathrm{p}>0,05)$.

Table 6.Relationship between daily meats intake and premature canities

\begin{tabular}{|c|c|c|c|c|c|c|c|}
\hline \multirow{3}{*}{ Variable } & \multicolumn{6}{|c|}{ Premature Canities } & \multirow{3}{*}{$P$ value } \\
\hline & \multicolumn{2}{|c|}{ Yes } & \multicolumn{2}{|c|}{ No } & \multicolumn{2}{|c|}{ Total } & \\
\hline & $\mathrm{n}$ & $\%$ & $\mathrm{n}$ & $\%$ & $\mathrm{~N}$ & $\%$ & \\
\hline \multicolumn{8}{|c|}{ Meats intake/daily } \\
\hline Yes & 26 & 45,6 & 7 & 30,4 & 33 & 41,3 & 0,319 \\
\hline No & 31 & 54,4 & 16 & 69,6 & 47 & 58,8 & \\
\hline Total & 57 & 100 & 23 & 100 & 80 & 100 & \\
\hline
\end{tabular}

Table 6 show that the most incidents of premature canities occurred to the respondents who had low daily meats intake as many as 31 people $(54,4 \%)$ while the respondents who had meats intake everyday as 26 people $(45,6 \%)$. Based on statistical tests, $\mathrm{p}$ value $=0,319$ was obtained so there was no a significant relationship between daily meats intake and premature canities ( $\mathrm{p}$ > $0,05)$. 
Table 7.Relationship between daily milks intake and premature canities

\begin{tabular}{|c|c|c|c|c|c|c|c|}
\hline \multirow{3}{*}{ Variable } & \multicolumn{6}{|c|}{ Premature Canities } & \multirow{3}{*}{$P$ value } \\
\hline & \multicolumn{2}{|c|}{ Yes } & \multicolumn{2}{|c|}{ No } & \multicolumn{2}{|c|}{ Total } & \\
\hline & $\mathrm{n}$ & $\%$ & $\mathrm{n}$ & $\%$ & $\mathrm{~N}$ & $\%$ & \\
\hline \multicolumn{8}{|c|}{ Milks intake/daily } \\
\hline Yes & 22 & 38,6 & 12 & 52,2 & 34 & 42,5 & 0,389 \\
\hline No & 35 & 61,4 & 12 & 47,8 & 46 & 57,5 & \\
\hline Total & 57 & 100 & 23 & 100 & 80 & 100 & \\
\hline
\end{tabular}

Table 7 show that the most incidents of premature canities occurred to the respondents who had low daily milks intake as many as 35 people $(61,4 \%)$ while the respondents who had milks intake everyday as 22 people $(38,6 \%)$. Based on statistical tests, $\mathrm{p}$ value $=0,389$ was obtained so there was no a significant relationship between daily milks intake and premature canities ( $p$ > $0,05)$.

Table 8.Relationship between comorbidities and premature canities

\begin{tabular}{|c|c|c|c|c|c|c|c|}
\hline \multirow{3}{*}{ Variable } & \multicolumn{6}{|c|}{ Premature Canities } & \multirow{3}{*}{$P$ value } \\
\hline & \multicolumn{2}{|c|}{ Yes } & \multicolumn{2}{|c|}{ No } & \multicolumn{2}{|c|}{ Total } & \\
\hline & $\mathrm{n}$ & $\%$ & $\mathrm{n}$ & $\%$ & $\mathrm{n}$ & $\%$ & \\
\hline \multicolumn{8}{|l|}{ Comorbities } \\
\hline None & 44 & 77,2 & 13 & 56,5 & 57 & 71,3 & \multirow{3}{*}{0,172} \\
\hline Anemia & 2 & 3,5 & 2 & 8,7 & 4 & 5,0 & \\
\hline Others & 11 & 19,3 & 8 & 34,8 & 28 & 35,0 & \\
\hline Total & 57 & 100 & 23 & 100 & 80 & 100 & \\
\hline
\end{tabular}

Table 8 show that in this study, premature canities occurred in the respondents without any comorbidities as 44 people $(77,2 \%)$, respondents who had anemia as 2 people $(3,5 \%)$, and respondents with others comorbidites as 11 people $(19,3 \%)$. Based on statistical tests, $\mathrm{p}$ value $=$ 0,172 was obtained so there was no a significant relationship between comorbidities and premature canities $(\mathrm{p}>0,05)$. 
Table 9.Relationship between smoking and premature canities

\begin{tabular}{lccccccc}
\hline \multirow{2}{*}{ Variable } & \multicolumn{6}{c}{ Premature Canities } & \multirow{2}{*}{ P value } \\
\cline { 2 - 7 } & \multicolumn{2}{c}{ Yes } & \multicolumn{2}{c}{ No } & \multicolumn{2}{c}{ Total } & \\
\cline { 2 - 7 } \multicolumn{1}{c}{ Smoking } & $\mathbf{n}$ & $\boldsymbol{\%}$ & $\mathbf{n}$ & $\boldsymbol{\%}$ & $\mathbf{N}$ & $\%$ & \\
Never & 56 & 98,2 & 23 & 100 & 79 & 98,7 & \multirow{2}{*}{1,00} \\
$1-5$ sticks/day & 1 & 1,8 & 0 & 0,0 & 1 & 1,3 & \\
\hline Total & 57 & 100 & 23 & 100 & 80 & 100 & \\
\hline
\end{tabular}

Table 9 show that in this study, the respondents with premature canities but never smoked as many as 56 people $(98,2 \%)$, while the group who consumed $1-5$ cigarettes/day as 1 person $(1,8 \%)$. Based on statistical tests, $\mathrm{p}$ value $=1,00$ was obtained so there was no a significant relationship between smoking and premature canities $(p>0,05)$.

Table 10.Relationship between alcohol consumption and premature canities

\begin{tabular}{|c|c|c|c|c|c|c|c|}
\hline \multirow{3}{*}{ Variable } & \multicolumn{6}{|c|}{ Premature Canities } & \multirow{3}{*}{$P$ value } \\
\hline & \multicolumn{2}{|c|}{ Yes } & \multicolumn{2}{|c|}{ No } & \multicolumn{2}{|c|}{ Total } & \\
\hline & $\mathbf{n}$ & $\%$ & $\mathbf{n}$ & $\%$ & $\mathbf{n}$ & $\%$ & \\
\hline \multicolumn{8}{|c|}{ Alcohol consumption } \\
\hline Never & 56 & 98,2 & 23 & 100 & 79 & 98,7 & 1,00 \\
\hline 1-2 times/month & 1 & 1,8 & 0 & 0,0 & 1 & 1,3 & \\
\hline Total & 57 & 100 & 23 & 100 & 80 & 100 & \\
\hline
\end{tabular}

Table 10 show that in this study, the respondents with premature canities but never consumed alcohol as many as 56 people $(98,2 \%)$, while the group who consumed alcohol 1-2 times/month as 1 person $(1,8 \%)$. Based on statistical tests, $\mathrm{p}$ value $=1,00$ was obtained so there was no a significant relationship between smoking and premature canities $(p>0,05)$.

Table 11.Relationship between stress and premature canities

\begin{tabular}{|c|c|c|c|c|c|c|c|}
\hline \multirow{3}{*}{ Variable } & \multicolumn{6}{|c|}{ Premature Canities } & \multirow{3}{*}{$\underset{\text { value }}{\mathbf{P}}$} \\
\hline & \multicolumn{2}{|c|}{ Yes } & \multicolumn{2}{|c|}{ No } & \multicolumn{2}{|c|}{ Total } & \\
\hline & $\mathbf{n}$ & $\%$ & $\mathbf{n}$ & $\%$ & $\mathbf{n}$ & $\%$ & \\
\hline Stress & & & & & & & 021 \\
\hline Mild & 4 & 7,0 & 7 & 30,4 & 11 & 13,8 & \\
\hline
\end{tabular}




\begin{tabular}{lllllll} 
Moderate & 42 & 73,7 & 12 & 52,2 & 54 & 67,5 \\
Sever & 11 & 19,3 & 4 & 17,4 & 15 & 18,8 \\
\hline Total & 57 & 100 & 23 & 100 & 80 & 100 \\
\hline
\end{tabular}

Table 11 show that in this study, the respondents with premature canities who had mild stress as 4 people $(7,0 \%)$, respondents who had moderate stress as 42 people $(73,7 \%)$, and the respondents who had severe stress as 11 people $(19,3 \%)$. Based on statistical tests, $\mathrm{p}$ value $=$ 0,021 was obtained so there was a significant relationship between smoking and premature canities $(\mathrm{p}<0,05)$.

Table 11. Logistic regression of multivariate analysis

\begin{tabular}{|c|c|c|c|c|c|c|c|c|c|c|}
\hline \multirow{3}{*}{$\begin{array}{l}\text { Independent } \\
\text { variables }\end{array}$} & \multirow{3}{*}{$\begin{array}{c}\text { Dependent } \\
\text { variables }\end{array}$} & \multicolumn{4}{|c|}{ Premature Canities } & \multirow{3}{*}{ Total } & \multirow{3}{*}{$\begin{array}{c}P \\
\text { value }\end{array}$} & \multirow{3}{*}{$\mathbf{O R}$} & \multicolumn{2}{|c|}{$95 \%$ CI } \\
\hline & & \multicolumn{2}{|c|}{ Yes } & \multicolumn{2}{|c|}{ No } & & & & Lower & Upper \\
\hline & & $\mathbf{n}$ & $\%$ & $\mathrm{n}$ & $\%$ & & & & & \\
\hline \multirow{4}{*}{$\begin{array}{l}\text { Family } \\
\text { History }\end{array}$} & Father & 23 & 40,4 & 4 & 17,4 & 27 & \multirow{4}{*}{0,008} & \multirow{4}{*}{11,69} & \multirow{4}{*}{2,498} & \multirow{4}{*}{54,732} \\
\hline & Mother & 12 & 21,1 & 0 & 0,0 & 12 & & & & \\
\hline & Sibling & 12 & 21,1 & 1 & 4,3 & 13 & & & & \\
\hline & None & 10 & 17,5 & 18 & 78,3 & 28 & & & & \\
\hline \multirow{3}{*}{ BMI } & Normoweight & 14 & 24,6 & 9 & 39,1 & 23 & \multirow{3}{*}{0,379} & \multirow{3}{*}{0,329} & \multirow{3}{*}{0,54} & \multirow{3}{*}{1,998} \\
\hline & Overweight & 19 & 33,3 & 10 & 43,5 & 29 & & & & \\
\hline & Obese & 24 & 42,1 & 4 & 17,4 & 28 & & & & \\
\hline \multirow{3}{*}{ Comorbidities } & None & 44 & 77,2 & 13 & 56,5 & 57 & \multirow{3}{*}{0,161} & \multirow{3}{*}{1,4} & \multirow{3}{*}{0,078} & \multirow{3}{*}{25,035} \\
\hline & Anemia & 2 & 3,5 & 2 & 8,7 & 4 & & & & \\
\hline & Others & 11 & 19,3 & 8 & 34,8 & 19 & & & & \\
\hline \multirow{3}{*}{ Stress } & Mild & 4 & 7,0 & 7 & 30,4 & 11 & \multirow{3}{*}{0,497} & \multirow{3}{*}{0,441} & \multirow{3}{*}{0,061} & \multirow{3}{*}{3,161} \\
\hline & Moderate & 42 & 73,7 & 12 & 52,2 & 54 & & & & \\
\hline & Severe & 11 & 19,3 & 4 & 17,4 & 15 & & & & \\
\hline
\end{tabular}

Table 11 show that in this study, the result of the logistic regression of multivariate analysis were obtained on all independent variables with $\mathrm{p}$ value $<0,25$, the most significant relationship was family history with $\mathrm{p}$ value $=0,008(\mathrm{p}$ value $<0,05)$ and $\mathrm{OR}=11,69$ so family history 12 times greater causing premature canities in students of Faculty of Medicine, Universitas Sumatera Utara.

\section{Discussion}

This study, according to a study conducted by Dautalabad, et al, in India 2016, the prevalence of premature canities was higher in women $(51,9 \%)$ than in men $(48,1 \%)$ [11]. Also the study 
conducted by Bhat et al, in 2016, revealed that the ratio of premature canities in men and women was 1:1 which means there is no relationship between gender and premature canities [12]. The results of the relationship between family history and premature canities are the same as the study conducted by Devaraj et al, in 2016 that there was a relationship between family history and premature canities (50.4\%) [2], as well as the study by Bhat et al, in 2016, that the parent's history who had premature canities was $42.6 \%$ and the sibling's history who had premature canities was $14.2 \%$ [12]. In a study conducted by Shin et al, in South Korea in 2015 found that there was a relationship between the father's history who had premature canities and the incidence of premature canities in respondents (33.3\%) as well as the mother's history and premature canities $(11.2 \%)[3]$.

There was no significant relationship between premature canities and BMI in respondents. This matched with the results of a study by Zayed et al, in Jordan 2013 and El-Seikh et al, in Egypt 2018 that reported that there was no significant association between premature canities and BMI [13] [14]. Shin H et al, in South Korea 2015 also found that premature canities was significantly higher in the overweight and obese groups than in the normal weight group [3].

Vegetables are a food source from iron, and in research conducted by Bhat et al in 2013 and Elseikh et al in 2018 explained that iron deficiency can affect the abnormality of pigmentation in hair [12] [14]. So iron levels and frequency of vegetable consumption are lower in people with premature canities. Meats are also the main food source of vitamin B12, and in the study conducted by Bhat et al in 2013 found out that vitamin B12 influences hair growth and pigmentation processes [12]. Hence, vitamin B12 levels and frequency of meat consumption are lower in people with premature canities. Milk consumption in respondents is related to calcium deficiency associated with the incidence of premature canities. In the study by Bhat et al, in 2013 and El-seikh et al, in 2018 explained that premature canities is often associated with calcium deficiency which affects the process of melanogenesis, so calcium levels and frequency of milk consumption are lower in people with premature canities [12] [14]. The results of the relationship between comorbidities and premature canities are the same as the study conducted by Naieni et al, in Iran 2011 that there was no relationship between comorbidities with the incidence of premature canities [10].

The results of the relationship between alcohol consumption and premature canities are the same as the study conducted by Belli et al, in Turkey 2016 that the respondents who had premature canities but never consumed alcohol was 79,1\% [8]. The results of the relationship between smoking and premature canities are the same as the study conducted by Shin et al, in South Korea 2016 that the prevalence of premature canities was higher in non-smoking group $(84,7 \%)$ than in the smoking group $(15,3 \%)$ [3]. The results of the relationship between stress 
and premature canities are the same as the study conducted by Belli et al, in 2016 that the incidence of premature canities was influenced by stress [8].

\section{Conclusion}

In conclusion, there is a correlation between premature canities and family history and stress in medical students of Universias Sumatera Utara class 2016-2018. There is no correlation between premature canities and gender, body mass index, diet, comorbidities, smoking, and alcohol consumptions in medical students of Universias Sumatera Utara class 2016-2018. Family history is the most dominant risk factors that associated to premature canities in medical students of University of Sumatera Utara class 2016-2018.

\section{Suggestion}

From this research, the researcher suggests that there will be health promotion regarding premature canities so the community, especially medical students of Universitas Sumatera Utara can be more aware of the risk factors associated to premature canities and can prevent the incidence. This research can be continued by looking for the relationship of other factors such as serum level of micronutrients and the level of achievement index on premature canities in medical students of Universitas Sumatera Utara.

\section{References}

[1] S. Mistry, J. R.Gosh, A. R. Bandyopadhyay, "Histomorphological and Quantitive Characteristics of Black and Gray Human Scalp Hair," Journal of Life Science, vol. 2, no. 1, pp. 49-52, 2010.

[2] Y. Devaraj et al., "A Clinicoepidemiology Study of Premature Canities of Degree College Students in The Rural Area," International Journal of Advance in Case Reports, vol. 3, no. 14, pp. 489-493, 2016.

[3] H. Shin et al, "Association of Premature Hair Graying with Family History, Smoking, and Obesity: A Cross-Sectional Study," Journal of the American Academy of Dermatology, vol. 72, no. 2, pp. 321-327, 2016.

[4] P. Ashraf, R. Simanulingkalit, N. K. Jusuf, "Association between Premature Canities and Quality of Life at Medan, Indonesia 2017," Bali Medical Journal, vol. 7, no.2, pp. 339345, 2018.

[5] R. G. Brown, K. Harmen, and G. Johnston, Lecture Notes: Dermatology, Wiley \& Sons, Ltd, 2016.

[6] P. K. Nigam, and P. Nigam, "Prematur Graying of Hair (Premature Canities): A Concern for Parent and Child," Journal of Pigmentary Disorder, vol.4, no.1, pp. 1-6, 2017.

[7] A. Kumar, H. Shamim, and U. Nagaraju, "Premature Graying of Hair: Review with Updates," International Journal of Trichology, vol. 10, no.5, pp. 198-203, 2018.

[8] A. A. Belli, F. Etgu, S. O. Gok, B. Kara, G. Dogan, "Risk Factor for Premature Hair Graying in Young Turkish Adults," Pediatric Dermatology, vol.33, no. 4, pp. 438-442, 2016. 
[9] S. Chakrabarty, P. G. Krishnappa, D. G. Gowda, J. Hiremath, "Factors Associated with Premature Hair Graying in Young Indian Population," International Journal of Dermatology, vol. 8, no.1, pp. 11-14, 2016.

[10] F. Naieni, B. Ebrahimi, H. R.Vakilian, Z. Shahmoradi, "Serum Iron, Zinc, and Copper Concentration in Premature Graying of Hair," Biological Trace Elements Research, vol. 146, no. 1, pp. 30-34, 2011.

[11] D. Daulatabad, A. Singal, C. Grover, N. Chhillar, "Profile of Indian Patients with Premature Canities," Indian Journal of Dermatology, Venereology, and Leproplogy, vol. 82, no. 2, pp. 169-172, 2016.

[12] Y. Bhat et al., "Serum Iron, Ferritin and Calcium Levels in Premature Canities," Hair: Therapy \& Transplantation, vol. 6, no.1, pp. 1-3, 2016.

[13] A. A. Zayed, A. D. Shahait, M. N. Ayoub, A. Yousef, "Smoker's hair: Does smoking cause premature hair graying?," Indian Dermatology Online Journal, vol.4, no.2, pp. 90-92, 2013.

[14] A. M. El-Sheikh, N. N. Elfar, H. A. Mourad, E. S. Hewedy, "Relationship between Trace Elements and Premature Hair Graying," International Journal of Trichology, vol. 10, no.6, pp.278-283, 2018. 